Research/Technical Note

\title{
A Useful Technique for Removing a Broken Guide-wire in the Femoral Neck Through the Proximal Femoral Nail Hole During Operation
}

\author{
Wenrui Wu, Simin Luo, Tengfeng Zhuang, Ning Liu, Zhengang Zha* \\ Department of Orthopaedics, The First Affiliated Hospital of Jinan University, Guangzhou, China \\ Email address: \\ zhzgg@vip.163.com (Zhengang Zha), ray510@163.com (Zhengang Zha) \\ ${ }^{*}$ Corresponding author
}

\section{To cite this article:}

Wenrui Wu, Simin Luo, Tengfeng Zhuang, Ning Liu, Zhengang Zha. A Useful Technique for Removing a Broken Guide-wire in the Femoral Neck Through the Proximal Femoral Nail Hole During Operation. Journal of Surgery. Vol. 7, No. 5, 2019, pp. 128-131.

doi: $10.11648 /$ j.js.20190705.13

Received: July 8, 2019; Accepted: August 10, 2019; Published: August 26, 2019

\begin{abstract}
Context: Cannulated instruments are usually used while operating around the hip joint for fractures. For example, femoral neck fracture, intertrochanteric fracture, etc. Perioperative instrument breakage such as kirschner wires and guide pins is not infrequent. The fragment of instruments must be removed in order to avoid injury some important organizations. If the fragment further migrate to the acetabulum can result in serious or fatal complications. Removing a broken guide-wire is difficult and challenging even for experienced surgeons. Case Report: We present a simple and useful method to remove the broken guide-wire in the hip joint just using cannulated reamer to ream over the guide wire under the C-arm image intensifier. At first, confirm the cannulated reamer to sight the broken end of the wire, and passed through the broken guide-wire. Then, further reaming with low speed rotation till the beginning of the reamer encased the broken guide-wire. The reamer was withdrawn a few millimeters, after confirmation of the broken guide-wire dislodgment and firmly engaged within the drill lumen by the C-arm image intensifier. Reverse the guide-wire slightly with the reamer through the Proximal Femoral Nail hole. Conclusions: With this method, the authors were able to remove the broken guide wire easily in a few minutes.
\end{abstract}

Keywords: Broken Guide-wire, Removing, Hip Joint, Cannulated Reamer

\section{Introduction}

Failure or breakage of orthopedic instruments such as Kirschner, drill bits and guide-wire during the operation is not an infrequent occurrence, and has been reported in various clinical settings [1-4]. The most commonly reported breaks of instruments during orthopedic procedures are drill bits, followed by Kirschner wires and cannulated guide-wire [3]. The breakage of instruments must be removed in order to avoid injury of the arteries and nerves, articular cartilage damage and consequent early degenerative arthritis if they are located near nerves, vessels, or joint surfaces [5-6]. If these further migrate to the acetabulum can lead to devastating and catastrophic complications. Removing a broken guide-wire is difficult and challenging even for experienced surgeons. We reports 2 cases of broken guide-wires during PFNA (proximal femoral nail antirotation, PFNA) placement for intertrochanteric fracture of the femur and discusses a new technique for removing the broken guide-wire quickly in the hip joint.

\section{Case Report}

Two patients with intertrochanteric fractures of the femur were admitted for PFNA fixation. The patients included a 79-year-old woman with a fracture of the right femur, a 71-year-old woman with a fracture of the left femur due to the accidental fall.

During the surgery, the patient was positioned supine on an extension table and underwent routine combined spinal-epidural anesthesia. Closed reduction of the 
intertrochanteric fracture was performed under a C-arm X-ray Machine. The surgeon performed a standard skin incision about $5 \mathrm{~cm}$ proximal from the tip of the greater trochanter and exposed. Then, the entry point was made. The guide wire was passed through and reaming of the canal by the rod. After reaming, assemble PFNA instruments, a proximal femoral nail with appropriate size and diameter was inserted easily. Choose aiming arm for PFNA blade insertion and prepare guide-wire insertion, position guide-wire with aiming device and insert it. However, when the cannulated reamer through the neck to the head of the femur, a sudden loss of resistance was unexpectedly encountered. On checking under a $\mathrm{C}$-arm image intensifier showed that the guide-wire was broken about $5 \mathrm{~cm}$ from the threaded end. In one case the broken guide-wire had not breached the hip joint, just touching the articular surface of the femoral head. In the other case the broken guide-wire was just protrusion into the hip joint. The surgeon therefore decided to remove it.

\section{Surgical Technique}

We tried to use the Kocher forceps and Kerrison/pituitary rongeur to remove the broken pin. The both above method for removing the broken guide-wire were failed. Ultimately, we used the original cannulated reamer to ream over the guide wire under the $\mathrm{C}$-arm image intensifier. At first, confirm the cannulated reamer to sight the broken end of the wire, and passed through the broken guide-wire in both image planes. Then, the reamer was advanced further reaming with low speed rotation till the beginning of the reamer encased the broken guide-wire. Afterwards, the reamer was withdrawn a few millimeters, compared anteroposterior and lateral view with all the previous images to confirm dislodgment of the guide-wire from its original position. After confirmation of the broken guide-wire dislodgment and firmly engaged within the drill lumen. Reverse the guide-wire slightly with the reamer from the bone. C-arm image intensifier confirmed complete removal of the guide-wire fragment. The PFNA blade was inserted in its original track and distal locking to complete the procedure The screw purchase was found to be satisfactory.

Postoperatively, the patient was started on quadriceps and knee exercises, mobilized with partial weight-bearing on the third postoperative day. All 2 patients were discharged after an uneventful recovery. Three months after operation, the patients fracture healing was satisfactory in all cases and the patient can walk independently without walking frame.

\section{Discussion}

Hardware like Kirschner/guide-wire/drill bit breakage during hip surgery can pose challenging and difficult problems for orthopedist. In most cases the site of breakage is deep within bone. In some cases, broken wire within a bone usually does not require removal, except it further migrate to the acetabulum, that can lead to devastating and catastrophic complications.
A major reason for guide-wire breakage due to repeated use $[2,5]$, which leads to decreasing mechanical strength and bending of these wires causes deformation. The guide-wires direction can also be deformed, when passing through bones of different densities (just like the hard subchondral bone of the femur and acetabulum), or when drilling at a low angle bending during insertion, or during transition through fracture area, when the traction is instable, or guide-wire deflection is crossing the joint space, what causes a minor change in direction. This change of direction may obstruct smooth passage of the reamer over the guide-wires and wear down the guide-wires, creating friction in the pin that results in breakage.

There are few reports in the literature about the methods for removing a broken guide-wire in hip joint [2, 6-8]. The known methods of removing the broken guide-wires include use of over-reaming, curette, pituitary forceps, arthroscopically assisted methods of retrieval and open arthrotomy and dislocation of the hip joint. Salunke et al [2] removal of all other implants. And enlarge the existing track using a cannulated drill bit. Then, used a $2 \mathrm{~mm}$ pituitary rongeur and Kerrison rongeur to grasp the tip of the broken guidewire and remove it. Arora et al [9] reported used a bone endoscopic assisted method for removing a broken guide-wire in hip joint. It requires a use of a special endoscope with a working canal and familiar with endoscopic operation, and maybe difficult to catch hold of a small protruding wire-tip by using graspers. Saurabh et al [10], reported used a grasping forceps through the proximal hole of recon nail to remove a broken guide wire transfixing the acetabulum, Koval et al, successfully used the method of a Kocher forceps or a needle holder aftermaking a wide entrance with dynamic hip screwreamer [11]. We tried using this method without any success. Graspers or Kocher forceps have a lack of strength to catch hold of a long segment broken guide-wire which have more holding power in bone, and retrieval it. Chouhan et al [12] reported used a "Push back" technique: A simple method to remove broken drill bit from the proximal femur. But its just suit for the broken end of the drill bit was protruding from the medial cortex of the femur.

Intrapelvic migration of broken guide-wires can be retrievaled with the assiste of laparoscopy, extraperitoneal or ilioinguinal approaches which may lead to significant complications like major vessels and/or nerves injury and increased morbidity [4]. Another method reported in the literature is using a dynamic hip screw reamer to ream the area surrounding the broken guide-wires for extraction easier [5], but may be lead to a large damages to the head of the femur in its weight bearing area. Mishra et al. pioneered the technique of making a window in the femoral neck to remove the broken wire was safe and effective [13], but may increased risk of femoral neck fracture and avascular necrosis of the femoral head.

The method, which we used in the present case that assist with the original cannulated reamer to remove the broken guide-wires with nail in situ and through the initial nail hole, 
avoid internal fixation loosening, bleeding complications, and shortening operation time, thus reduce the postoperative complications.

The operator only needs to continue the original operation procedures. Put the original cannulated reamer into the screw hole, confirm the reamer to sight the broken end of the wire, and pass through the broken guide-wire under a $\mathrm{C}$-arm image intensifier in both anteroposterior and lateral views. Because the cross-sectional shape of the pin changes to an oval rather than a round shape at the point of the break, reaming with a cannulated reamer engages the pin, allowing the pin to be removed easily [14]. Generally speaking, once the threads were engaged the broken piece of wire was easily removed by withdrawing the reamer. After our experience with the first patient, in the other 2 cases we used an original cannulated reamer to avoid any extensive reaming and trauma to the tissues. With this method, we were able to remove the broken pin easily in a few minutes.

The idea came out during our ordinary practice, sometimes the guide-wire may along with the reamer when withdraw the cannulated reamer. The guide-wire sometimes trapped, together with bone debris, within the lumen of the cannulated reamer, This was perhaps the reason why we could use the cannulated reamer to retrieve the broken guide-wire, and it also can use a cannulated drill bit to retrieve the broken guide-wire.

\section{Conclusions}

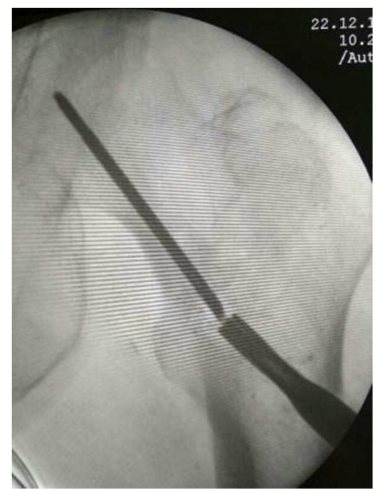

Figure 1. Broken pin in a 71-year-old patient at the time of reaming.

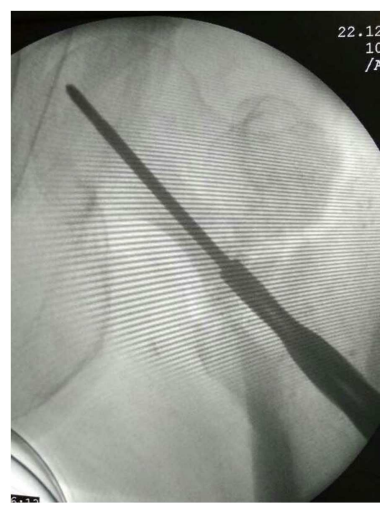

Figure 2. Confirm the cannulated reamer to sight the broken end of the wire.
Most of these breakage can be avoided. Furthermore, surgeons must have more patience while managing such problems during the operation. We present a useful and safe technique which simplifies the removal of broken guide-wire. The method we described was useful and worth alternative to retrieve broken guide-wire. and the method may be adapted to other sites as well.

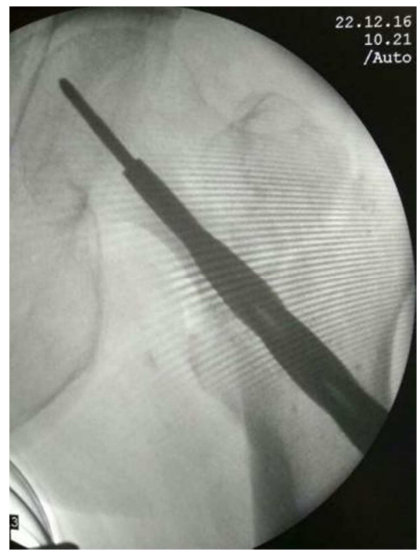

Figure 3. The reamer was passed through the broken guide-wire under the C-arm image intensifier.

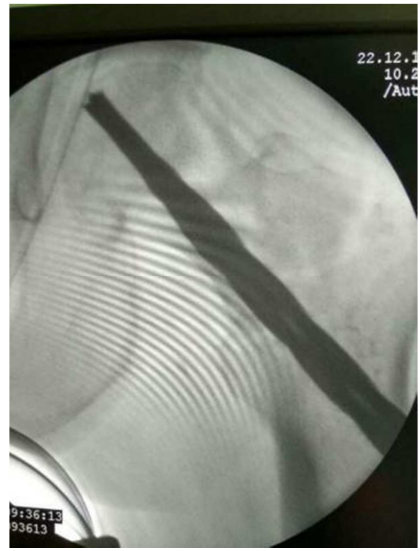

Figure 4. The reamer was advanced further reaming till its encased a large portion of the broken guide-wire. Afterwards, reverse the guide-wire with the reamer from the bone.

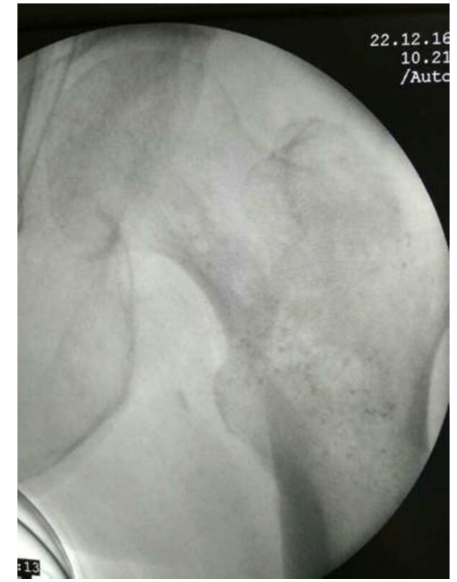

Figure 5. The broken pin is complete removed by a cannulated instrument. 


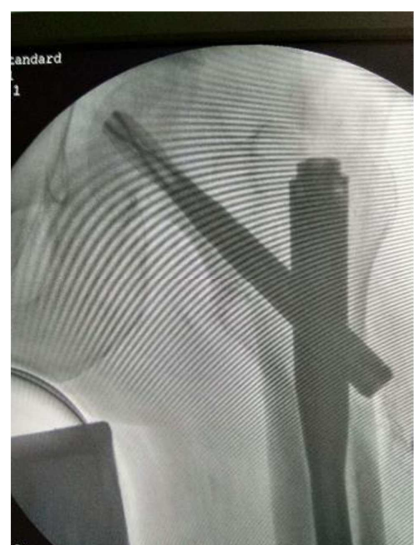

Figure 6. The PFNA blade was inserted in its original track to complete the procedure.

\section{References}

[1] Saini U C, Gopinathan N R, Aggarwal S, et al. Intrapelvic Protrusion of a Broken Guide Wire Fragment during Fixation of a Femoral Neck Fracture. The archives of bone and joint surgery. 2018, 6 (2): 155-156.

[2] Salunke, A. A., Menon, P. H., Nambi, G. I, et al. Removing a broken guidewire in the hip joint: treatment options and recommendations for preventing an avoidable surgical catastrophe. A case report. 2015. Sao Paulo Medical Journal, 133 (6), 531-534.

[3] Pichler W, Mazzurana P, Clement H, et al. Frequency of instrument breakage during orthopaedic procedures and its effects on patients. J Bone Joint Surg Am 90: 2652-2654, 2008.

[4] Sen RK, Tripathy SK, Aggarwal S, et al. Broken Kirschner or guide-wire retrieval: a report of 4 cases. Hip Int 20: 551-554, 2010 .
[5] Sharma H, Chauhan M, Maini L. A technique to remove a broken guide wire transfixing the hip joint. Acta Orthop Belg. 2008; 74 (5): 683-685.

[6] Sayegh FE, Tsintzas D, Kapetanos GA. Intrapelvic migration of a guide pin during fixation of a hip fracture: who and what is to blame? Acta Orthop Belg. 2005; 71 (2): 239-241.

[7] Sen RK, Tripathy SK, Aggarwal S, et al. Broken Kirschner or guide-wire retrieval: a report of 4 cases. Hip Int 2010; 20 (4): 551-4.

[8] Kumar A, Jain A, Saurabh S, et al. Technical tip for removal of broken cephalomedullary reamer tip in cases of proximal femoral nailing. Journal of clinical orthopaedics and trauma. 2019.

[9] Arora S, Maini L, Aggarwal V, et al. Broken guide-wire protruding into the hip joint: A bone endoscopicassisted retrieval method. Indian J Orthop 2012; 46: 109-12.

[10] Saurabh Jain, Abhishek Pathak, Rajeev Kant Pandey. Retrieval of a broken guide-wire transfixing the hip through the proximal femoral nail hole. Chinese Journal of Traumatology. 50. 2014; 17 (1): 50-53.

[11] Koval KJ, Zuckerman JD. Hip fractures. A practical guide to management [M]. New York: Springer, 2000: 20-1.

[12] Chouhan D K, Sharma S. "Push back" technique: A simple method to remove broken drill bit from the proximal femur. World journal of orthopedics. 2015, 6 (10): 847-849.

[13] Mishra P, Gautam VK. Broken guide wire with intrapelvic protrusion: a technique for removal. Injury. 2004; 35 (12): 1324-1326.

[14] Peivandi MT, Kachooei AR, Nazemian Z. New method to remove a broken guide pin in the hip joint. Orthopedics. 2011; 34 (10): e685-7. 